\title{
Derivatives of guanidine-based DNA minor groove binders as antiprotozoal agents
}

\author{
Adeyemi Rahman ${ }^{1^{*}}$, Isabel Rozas ${ }^{1}$
}

${ }^{1}$ School of Chemistry, TBSI, Trinity College Dublin, 152-160 Pearse St., Dublin 2

*Correspondence author: rahmanad@tcd.ie

\section{Introduction}

Parasitic infectious diseases such as malaria, human African trypanosomiasis (HAT) and leishmaniasis are a major concern in developing countries. According to the WHO, in 2018, there were 228 million cases of malaria with death rates reaching 405,000.1 In addition, in sub-Saharan Africa, HAT has a risk population of 65 million people and almost 1 million annual cases of leishmaniasis exist. ${ }^{2}$ Current standard of care for malaria includes drugs such as quinolones (e.g. chloroquine), anti-folates (e.g. pyrimethamine) or artemisinin. However, because emergence of multi-drug resistant protozoans, the identification of new drugs as well as new

therapeutic targets is needed. Compounds

that bind into the DNA minor-groove

(MGBs) are very promising for the

treatment of diseases at the DNA level.

俉

distamycin-A and netropsin are used to treat

parasitic diseases. 1,2

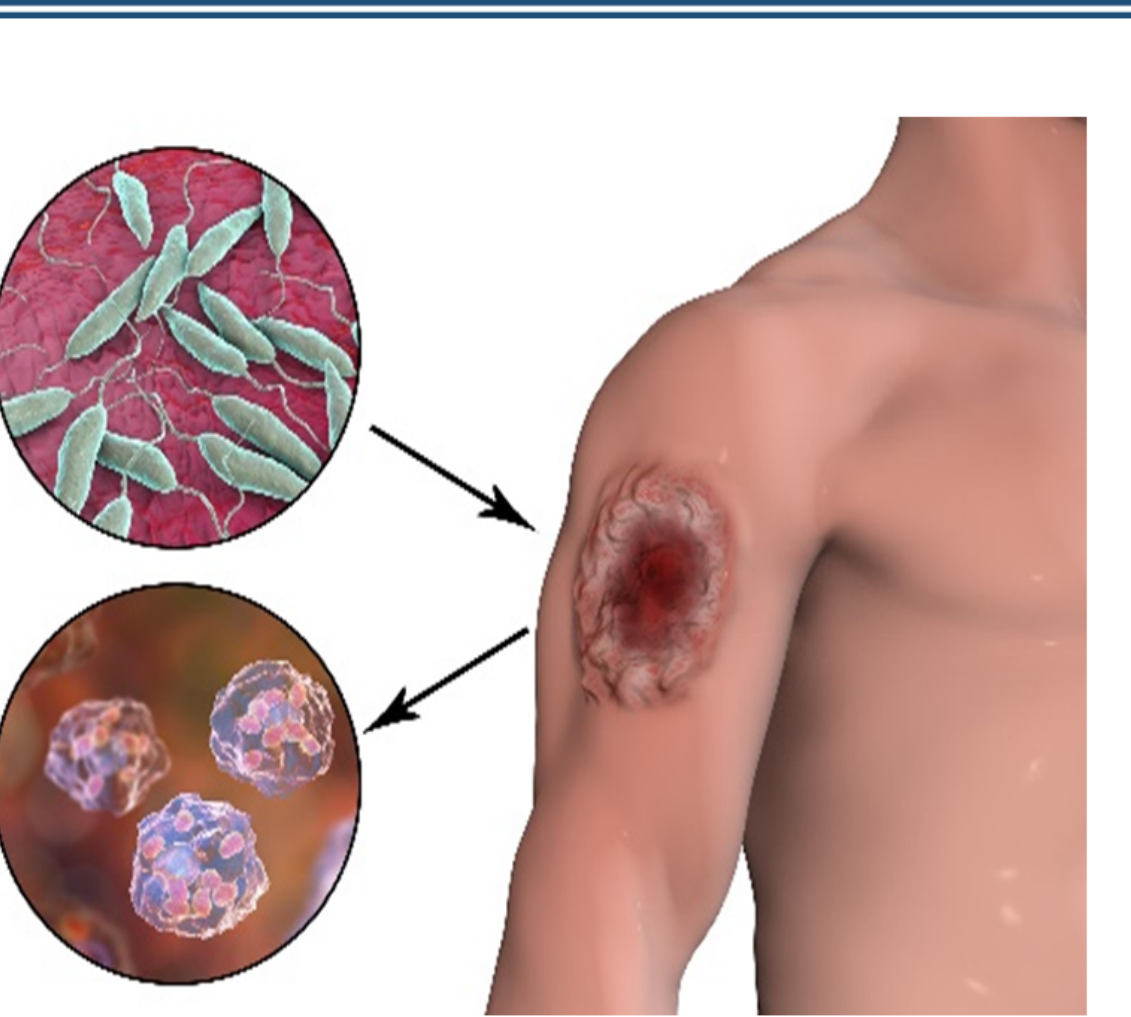

\section{Objectives}

Our lab has prepared a large number of symmetric and asymmetric dications that are strong MGBs. ${ }^{3,5}$ Recently, a series of amino alkyl derivatives of di-aromatic guanidines with very promising antimalarial activity were reported by us. ${ }^{4}$ Based on these results, a novel series of amino-alkyl derivatives were designed. Hence, ou objective is to computationally and biophysically study the interaction of these molecules with DNA and assess their ability as antiprotozoal agents.
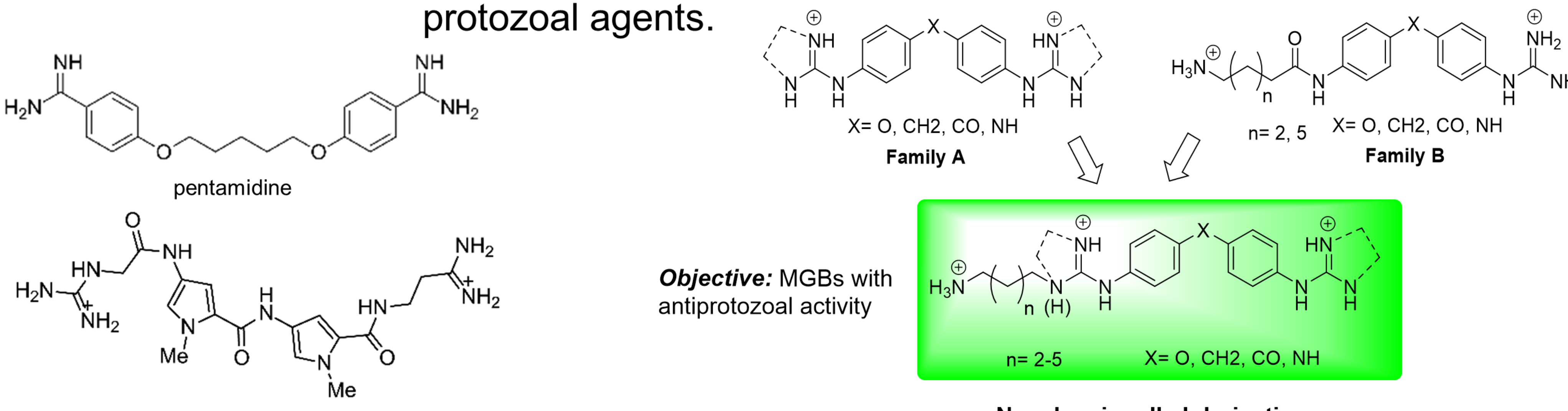

Novel aminoalkyl derivatives

netropsin

of diaryl bisguanidine/2-aminoimidazolines



